\title{
Direct synthesis of barium titanium oxyhydride promising for electrode material with hydrogen permeability
}

Tasuku Uchimura, ${ }^{\text {ab }}$ Fumitaka Takeiri, ${ }^{\text {abc }}$ Kei Okamoto, ${ }^{\text {ab }}$ Takashi Saito ${ }^{\text {de }}$, Takashi Kamiyama $^{\text {de }}$, and Genki Kobayashi*ab

${ }^{a}$ Department of Materials Molecular Science, Institute for Molecular Science, 38 Nishigo-naka, Myodaiji, Okazaki, Aichi 444-8585, Japan.

${ }^{b}$ SOKENDAI (The Graduate University for Advanced Studies), 38 Nishigo-naka, Myodaiji, Okazaki, Aichi 444-8585, Japan.

'PRESTO, Japan Science and Technology Agency (JST), Kawaguchi, Saitama 332-0012, Japan.

${ }^{d}$ Department of Materials Structure Science, High Energy Accelerator Research Organization (KEK), 2-4 Shirakata, Tokai, Naka, Ibaraki, 319-1106, Japan.

${ }^{e}$ SOKENDAI (The Graduate University for Advanced Studies), 2-4 Shirakata, Tokai, Naka, Ibaraki, 319-1106, Japan.

"Corresponding author (E-mail): gkobayashi@ims.ac.jp 


\begin{abstract}
Barium titanium oxyhydride $\mathrm{BaTiO}_{3-x} \mathrm{H}_{x}$ is a promising functional material that exhibits $\mathrm{H}^{-}$ $/ \mathrm{e}^{-}$mixed conduction and catalytic activity. Here we firstly report a direct synthesis of $\mathrm{BaTiO}_{3-x} \mathrm{H}_{x}$ by a mechanochemical method. The prepared polycrystalline sample was able to work as electrodes with hydrogen permeability.
\end{abstract}

\title{
Introduction
}

Oxyhydrides, wherein hydride ions $\left(\mathrm{H}^{-}\right)$stabilized in oxide frameworks, have recently become a new topic of materials science. Representative examples are the first case of transition-metal oxyhydride $\mathrm{LaSrCoO}_{3} \mathrm{H}_{0.7},{ }^{1}$ unusual pnictide superconductor LaFeAsO ${ }_{1-x} \mathrm{H}_{x},{ }^{2,3}$ and pure $\mathrm{H}^{-}$conductor $\mathrm{La}_{2-x-y} \mathrm{Sr}_{x+y} \mathrm{LiH}_{1-x+y} \mathrm{O}_{3-y}{ }^{4}$ In particular, titanium perovskite $\mathrm{BaTiO}_{3-x} \mathrm{H}_{x}$ is an important piece that exhibits metallic electronic conduction and high anion exchangeability. ${ }^{5-7}$ Such properties afford valuable chemical functions represented by catalytic activity for $\mathrm{NH}_{3}$ synthesis and $\mathrm{CO}_{2}$ reduction. ${ }^{8}{ }^{8}$ Furthermore, the $\mathrm{H}^{-} / \mathrm{e}^{-}$mixed conducting nature should be promising for the use of electrodes capable of hydrogen transmission in electrochemical devices for chemical/energy conversion.

The polycrystalline samples of $A \mathrm{TiO}_{3-x} \mathrm{H}_{x}(A=\mathrm{Ba}, \mathrm{Sr}, \mathrm{Ca}, \mathrm{Eu})$ have been previously prepared by solid-state topochemical reactions, in which a mixture of the corresponding 
oxide precursors $A \mathrm{TiO}_{3}$ and calcium hydride $\left(\mathrm{CaH}_{2}\right)$ are heated under vacuum. ${ }^{5,10,11}$ The hydrogen concentration $x$ can be varied depending on the reaction conditions including sintering temperature and period. However, controlling $x$ value, that is a critical factor for the abovementioned chemical functions, is still a challenge. It is because that not only thermodynamic but also kinetic factor, such as anion diffusivity in the lattice, should be considered. ${ }^{12,13}$ For example, $\mathrm{BaTiO}_{3-x} \mathrm{H}_{x}$ in single phase was obtained from the precursors of nano size $(\sim 170 \mathrm{~nm})$ particles, while the multi-phases with different hydrogen concentrations generated from those of larger particles $(10-30 \mu \mathrm{m}) .{ }^{10}$ In addition, the long reaction period for a week and washing process to remove the residual $\mathrm{CaH}_{2}$ and the byproduct $\mathrm{CaO}$ are inevitable. Such a complex and multi-step process might result in less reproducibility of functional performance and be unsuitable for future applicable use.

Mechanochemical synthesis through dry milling without heating can use mechanical energy for chemical reactions. Especially among research fields of catalyst and battery, it has become one of the effective methods for materials synthesis. For instance, mechanochemical prepared $\mathrm{CaO}-\mathrm{ZnO}$ mixed oxides show more catalytic activity for biodiesel production compared to those prepared by the coprecipitation method ${ }^{14} \cdot \mathrm{Li}_{2} \mathrm{~S}-\mathrm{P}_{2} \mathrm{~S}_{5}$ glass-ceramics exhibits high ionic conductivity at room temperature. ${ }^{15,16}$ Herein, we show a direct preparation of $\mathrm{BaTiO}_{3-x} \mathrm{H}_{x}$ by only 90 minutes of ball-milling, which is the first report of mechanochemical synthesis of oxyhydrides. 


\section{Results and Discussion}

Mechanochemical synthesis of polycrystalline samples of $\mathrm{BaTiO}_{3-x} \mathrm{H}_{x}$ were performed as below. Commercial reagents of $\mathrm{BaH}_{2}, \mathrm{BaO}, \mathrm{TiO}_{2}$, and $\mathrm{TiH}_{2}$ were used as starting materials. Those reagents were mixed in an Ar-filled glovebox using an agate mortar, following equation (1) with the nominal composition of $x_{\text {nom }}=0.1,0.25,0.5,1$.

$$
x \mathrm{BaH}_{2}+(1-x) \mathrm{BaO}+\mathrm{TiO}_{2} \rightarrow \mathrm{BaTiO}_{3-x} \mathrm{H}_{x}+0.5 x \mathrm{H}_{2}
$$

For the nominal composition of $x_{\text {nom }}=2, \mathrm{TiH}_{2}$ was used instead of $\mathrm{BaH}_{2}$ and $\mathrm{TiO}_{2}$ as following the equation (2).

$$
\mathrm{BaO}+\mathrm{TiH}_{2} \rightarrow \mathrm{BaTiOH}_{2}
$$

The mixtures of $1 \mathrm{~g}$ were sealed in a $\mathrm{ZrO}_{2}$ container together with $\mathrm{ZrO}_{2}$ balls. Mechanical activation was performed at 600,800 , and $1100 \mathrm{rpm}$ for $90 \mathrm{~min}$ without intervals using highenergy ball milling equipment (Emax, Retsch).

Figure 1 shows laboratory X-ray diffraction patterns of $x_{\text {nom }}=0.5$ samples with various milling conditions. For the sample after hand-milling, only profiles derived from the raw materials of $\mathrm{BaO}, \mathrm{BaH}_{2}$, and $\mathrm{TiO}_{2}$ were detected. After ball-milling at $600 \mathrm{rpm}$, the peaks were broadened, and new peaks appeared at $800 \mathrm{rpm}$ with a relatively high background. In contrast, at $1100 \mathrm{rpm}$, clear peaks indexable with a cubic unit cell of $a=4.0252 \AA$ were observed without any other 
peaks, indicating a formation of simple perovskite in a single phase. The powder color changed from white to black, suggesting the reduced partial $\mathrm{Ti}^{4+}$ to $\mathrm{Ti}^{3+}$, and the obtained cubic phase was stable in air. Those features are the same with those for previous reported $\mathrm{BaTiO}_{3-x} \mathrm{H}_{x}$ prepared by topochemical reaction using $\mathrm{CaH}_{2}{ }^{5}$ Thermal desorption spectroscopy (TDS) measurement was performed to check the presence of hydrogen in the product. Figure 2 (inset) shows the obtained spectrum corresponding to desorbed $\mathrm{H}_{2}$ molecule $(\mathrm{m} / \mathrm{z}=2)$ upon heating. The desorption gradually occurred above $200{ }^{\circ} \mathrm{C}$ and sharply increased around $480{ }^{\circ} \mathrm{C}$. The total amount of hydrogen overall temperature range was estimated by integrating the peak area, giving the hydrogen content $x$ of 0.427 for $\mathrm{BaTiO}_{3-x} \mathrm{H}_{x}$. Scanning electron microscopy (SEM) image of the obtained powder indicates a broad particle size distribution of 0.1-2 $\mu \mathrm{m}$ (Fig. S1), possibly inducing the $\mathrm{H}_{2}$ desorption at a wide range of temperatures.

The crystal structure and composition of the $x_{\text {nom }}=0.5$ product were estimated by Rietveld refinement. Powder synchrotron X-ray and neutron diffraction (SXRD and ND) data collected at room temperature were refined using RIETAN-FP and Z-Rietveld program, respectively. ${ }^{17,18} \mathrm{ND}$ refinement assuming a cubic perovskite $\mathrm{BaTiO}_{3-x} \mathrm{H}_{x}$ converged well and gave $x=0.3900(14)$ (Fig. 2 and Table S1). The estimated composition is consistent with both the SXRD refinement yielding a sizable amount of anion vacancy of $\delta=0.423(4)$ for $\mathrm{BaTiO}_{3-\delta}$ (Fig. S2 and Table S2) and the TDS result. A slight decrease of hydrogen from the nominal composition $\left(x_{\text {nom }}=0.5\right)$ could be 
derived from oxygen contamination in the raw material $\mathrm{BaH}_{2}$ or/and during milling using a $\mathrm{ZrO}_{2}$ pot and balls. From these results, we conclude that barium titanium oxyhydride $\mathrm{BaTiO}_{3-x} \mathrm{H}_{x}$ was successfully synthesized by mechanochemical reaction without significant hydrogen loss.

Laboratory XRD patterns of $x_{\text {nom }}=0.1,0.25,0.5,1$, and 2 after ball-milling at $1100 \mathrm{rpm}$ are summarized in Fig. 3a, both showing a formation of cubic perovskites. Byproducts mainly indexed for $\mathrm{BaH}_{2}$ appeared in $x_{\text {nom }}=2$. As $x_{\text {nom }}$ increases, the cubic profiles were shifted toward lower angles, e.g. 112 peaks highlighted in Fig. 3b. Figure 3c plots the calculated lattice volumes

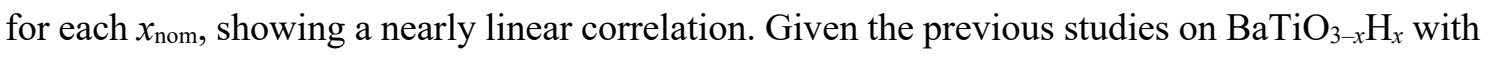
a roughly positive correlation between the lattice constants and $x$, the observed trend indicates that hydrogen concentration in mechanochemically prepared oxyhydrides could be tuned by appropriate setting of nominal compositions. The lattice constant of $4.0384 \AA$ for $x_{\text {nom }}=2$ is somewhat larger than that for $\mathrm{BaTiO}_{2.40} \mathrm{H}_{0.60}(4.0321 \AA)$ that is the most hydrogen-exchanged composition prepared by $\mathrm{CaH}_{2}$ reaction, ${ }^{5}$ possibly implying that the mechanochemical method could expand the hydrogen solubility limit in the titanium oxyhydrides.

In order to evaluate the electrode property for $\mathrm{BaTiO}_{3-x} \mathrm{H}_{\mathrm{x}}\left(x_{\text {nom }}=0.5\right)$, we constructed a symmetric cell where a $\mathrm{H}^{-}$conductor $\mathrm{LaSrLiH}_{2} \mathrm{O}_{2}$ sandwiched by $\mathrm{BaTiO}_{3-x} \mathrm{H}_{x}$, and performed electrochemical impedance spectroscopy (EIS) measurements under $\mathrm{H}_{2}$ flow in the temperature range of $150-325{ }^{\circ} \mathrm{C}$. The electrolyte $\mathrm{LaSrLiH}_{2} \mathrm{O}_{2}$ was prepared by sintering under ambient 
pressure, according to the previous report. ${ }^{19}$ The cell was prepared by sintering a three-layered pellet composed with $\mathrm{BaTiO}_{3-x} \mathrm{H}_{x}\left|\mathrm{LaSrLiH}_{2} \mathrm{O}_{2}\right| \mathrm{BaTiO}_{3-x} \mathrm{H}_{x}$ at $400{ }^{\circ} \mathrm{C}$ under uniaxial pressure of $300 \mathrm{MPa}$. Figure 4a presents a typical impedance spectrum obtained at $325^{\circ} \mathrm{C}$, composed of at least three semicircles that can be attributed to responses from the sum of bulk and grain boundary ("bulk+gb"), electrolyte/electrode interface ("interface"), and electrode, respectively at high-, middle-, and low-frequency regions. The spectrum was analyzed by fitting with the equivalent circuit shown in Fig. 4a, and an acceptable fit was obtained. The validity of this model was also confirmed by analyzing the spectrum of another cell with a thicker electrolyte using the same equivalent circuit (Fig. S3).

Temperature dependence of $\mathrm{H}^{-}$conductivity of the electrolyte $\left(\mathrm{LaSrLiH}_{2} \mathrm{O}_{2}\right)$ estimated from the bulk and grain boundary resistance is comparable to reported one (grey dashed line) using deposited $\mathrm{Au}$ as hydrogen blocking electrodes,${ }^{19}$ as shown in Fig. $4 \mathrm{~b}$. Notably, the pressed polycrystalline $\mathrm{BaTiO}_{3-\mathrm{x}} \mathrm{H}_{\mathrm{x}}$ sintered at a relatively low temperature $\left(400{ }^{\circ} \mathrm{C}\right)$ exhibits an electronic conductivity enough for electrode use. The activation energy for ion diffusion was estimated as $66.9 \mathrm{~kJ} / \mathrm{mol}$, which is smaller than the reported one of $80.7 \mathrm{~kJ} / \mathrm{mol}$, probably derived from the reduced grain boundary resistance owing to hot pressing. Furthermore, observation of the clear semicircle corresponding to electrode response, i.e., hydrogen charge transfer $\left(\mathrm{H}_{2}+2 \mathrm{e}^{-} \Leftrightarrow 2 \mathrm{H}^{-}\right)$, is similar to the EIS measurement of $\mathrm{H}^{-}$-conducting $\mathrm{BaH}_{2}$ with hydrogen permeable Pd film 
electrodes. ${ }^{20}$ This result firstly and clearly shows a capability of $\mathrm{BaTiO}_{3-\mathrm{x}} \mathrm{H}_{\mathrm{x}}$ as a hydrogenpermeable electrode derived from its $\mathrm{H}^{-} / \mathrm{e}^{-}$mixed conducting property. The relatively large and broad semicircle for the electrolyte/electrode interfacial resistance might reflect the intrinsic complexity of the heterointerface of the powder samples, including the contact area and compositional distribution.

\section{Conclusion}

In conclusion, we have succeeded in direct synthesis of barium titanium oxyhydride $\mathrm{BaTiO}_{3-x} \mathrm{H}_{x}$ by mechanochemical reaction, allowing gram-scale and speedy preparation of the functional material. This is the first case of mechanochemical synthesis of oxyhydrides and should be applied for other known compounds. Furthermore, the reaction condition without external heating might offer a route to new metastable oxyhydrides. It is also confirmed that the prepared polycrystalline $\mathrm{BaTiO}_{3-\mathrm{x}} \mathrm{H}_{\mathrm{x}}$ worked as a hydrogen-permeable electrode. This result suggests that $\mathrm{H}^{-} / \mathrm{e}^{-}$mixed conducting oxyhydrides are promising electrodes in electrochemical chemical/energy conversion devices.

\section{Acknowledgement}


This work was supported by JSPS, KAKENHI (Grants No. 18H05516, 18H02066, 19K15661, 20H02828) and JST, PRESTO (JPMJPR20T2). Synchrotron and neutron radiation experiments were approved by JASRI (2019A1084, 2020A1659) and IMSS, KEK (2019S10).

\section{References}

1 M. A. Hayward, E. J. Cussen, J. B. Claridge, M. Bieringer, M. J. Rosseinsky, C. J. Kiely, S. J. Blundell, I. M. Marshall and F. L. Pratt, Science (80-. )., 2002, 295, 1882-1884.

2 S. Iimura, S. Matuishi, H. Sato, T. Hanna, Y. Muraba, S. W. Kim, J. E. Kim, M. Takata and H. Hosono, Nat. Commun., , DOI:10.1038/ncomms 1913.

3 M. Hiraishi, S. Iimura, K. M. Kojima, J. Yamaura, H. Hiraka, K. Ikeda, P. Miao, Y. Ishikawa, S. Torii, M. Miyazaki, I. Yamauchi, A. Koda, K. Ishii, M. Yoshida, J. Mizuki, R. Kadono, R. Kumai, T. Kamiyama, T. Otomo, Y. Murakami, S. Matsuishi and H. Hosono, Nat. Phys., 2014, 10, 300-303.

4 G. Kobayashi, Y. Hinuma, S. Matsuoka, A. Watanabe, M. Iqbal, M. Hirayama, M. Yonemura, T. Kamiyama, I. Tanaka and R. Kanno, Science (80-. )., 2016, 351, 1314-1317.

5 Y. Kobayashi, O. J. Hernandez, T. Sakaguchi, T. Yajima, T. Roisnel, Y. Tsujimoto, M. Morita, Y. Noda, Y. Mogami, A. Kitada, M. Ohkura, S. Hosokawa, Z. Li, K. Hayashi, Y. Kusano, J. E. Kim, N. Tsuji, A. Fujiwara, Y. Matsushita, K. Yoshimura, K. Takegoshi, M. Inoue, M. Takano and H. Kageyama, Nat. Mater., 2012, 11, 507-511.

6 T. Yajima, F. Takeiri, K. Aidzu, H. Akamatsu, K. Fujita, W. Yoshimune, M. Ohkura, S. Lei, V. Gopalan, K. Tanaka, C. M. Brown, M. A. Green, T. Yamamoto, Y. Kobayashi and H. Kageyama, Nat. Chem., 2015, 7, 1017-1023.

7 N. Masuda, Y. Kobayashi, O. Hernandez, T. Bataille, S. Paofai, H. Suzuki, C. Ritter, N. Ichijo, Y. Noda, K. Takegoshi, C. Tassel, T. Yamamoto and H. Kageyama, J. Am. Chem. Soc., 2015, 137, 15315-15321.

8 Y. Tang, Y. Kobayashi, N. Masuda, Y. Uchida, H. Okamoto, T. Kageyama, S. Hosokawa, F. Loyer, K. Mitsuhara, K. Yamanaka, Y. Tamenori, C. Tassel, T. Yamamoto, T. Tanaka and H. Kageyama, Adv. Energy Mater., , DOI:10.1002/aenm.201801772.

9 Y. Tang, Y. Kobayashi, C. Tassel, T. Yamamoto and H. Kageyama, Adv. Energy Mater., , DOI:10.1002/aenm.201800800.

10 T. Sakaguchi, Y. Kobayashi, T. Yajima, M. Ohkura, C. Tassel, F. Takeiri, S. Mitsuoka, H. Ohkubo, T. Yamamoto, J. E. Kim, N. Tsuji, A. Fujihara, Y. Matsushita, J. Hester, M. Avdeev, K. Ohoyama and H. Kageyama, Inorg. Chem., 2012, 51, 11371-11376. 
11 T. Yamamoto, R. Yoshii, G. Bouilly, Y. Kobayashi, K. Fujita, Y. Kususe, Y. Matsushita, K. Tanaka and H. Kageyama, Inorg. Chem., 2015, 54, 1501-1507.

12 F. Takeiri, T. Yajima, T. Yamamoto, Y. Kobayashi, T. Matsui, J. Hester and H. Kageyama, J. Solid State Chem., 2017, 256, 33-37.

13 F. Takeiri, K. Aidzu, T. Yajima, T. Matsui, T. Yamamoto, Y. Kobayashi, J. Hester and H. Kageyama, Inorg. Chem., 2017, 56, 13035-13040.

14 Ž. Kesić, I. Lukić, D. Brkić, J. Rogan, M. Zdujić, H. Liu and D. Skala, Appl. Catal. A Gen., 2012, 427-428, 58-65.

15 A. Hayashi, S. Hama, T. Minami and M. Tatsumisago, Electrochem. commun., 2003, 5, $111-114$.

16 H. Yamane, M. Shibata, Y. Shimane, T. Junke, Y. Seino, S. Adams, K. Minami, A. Hayashi and M. Tatsumisago, Solid State Ionics, 2007, 178, 1163-1167.

17 F. Izumi and K. Momma, in Solid State Phenomena, Trans Tech Publ, 2007, vol. 130, pp. $15-20$.

18 R. Oishi, M. Yonemura, Y. Nishimaki, S. Torii, A. Hoshikawa, T. Ishigaki, T. Morishima, K. Mori and T. Kamiyama, Nucl. Instruments Methods Phys. Res. Sect. A Accel. Spectrometers, Detect. Assoc. Equip., 2009, 600, 94-96.

19 A. Watanabe, G. Kobayashi, N. Matsui, M. Yonemura, A. Kubota, K. Suzuki, M. Hirayama and R. Kanno, Electrochemistry, 2017, 85, 88-92.

20 M. C. Verbraeken, C. Cheung, E. Suard and J. T. S. Irvine, Nat. Mater., 2015, 14, 95-100. 


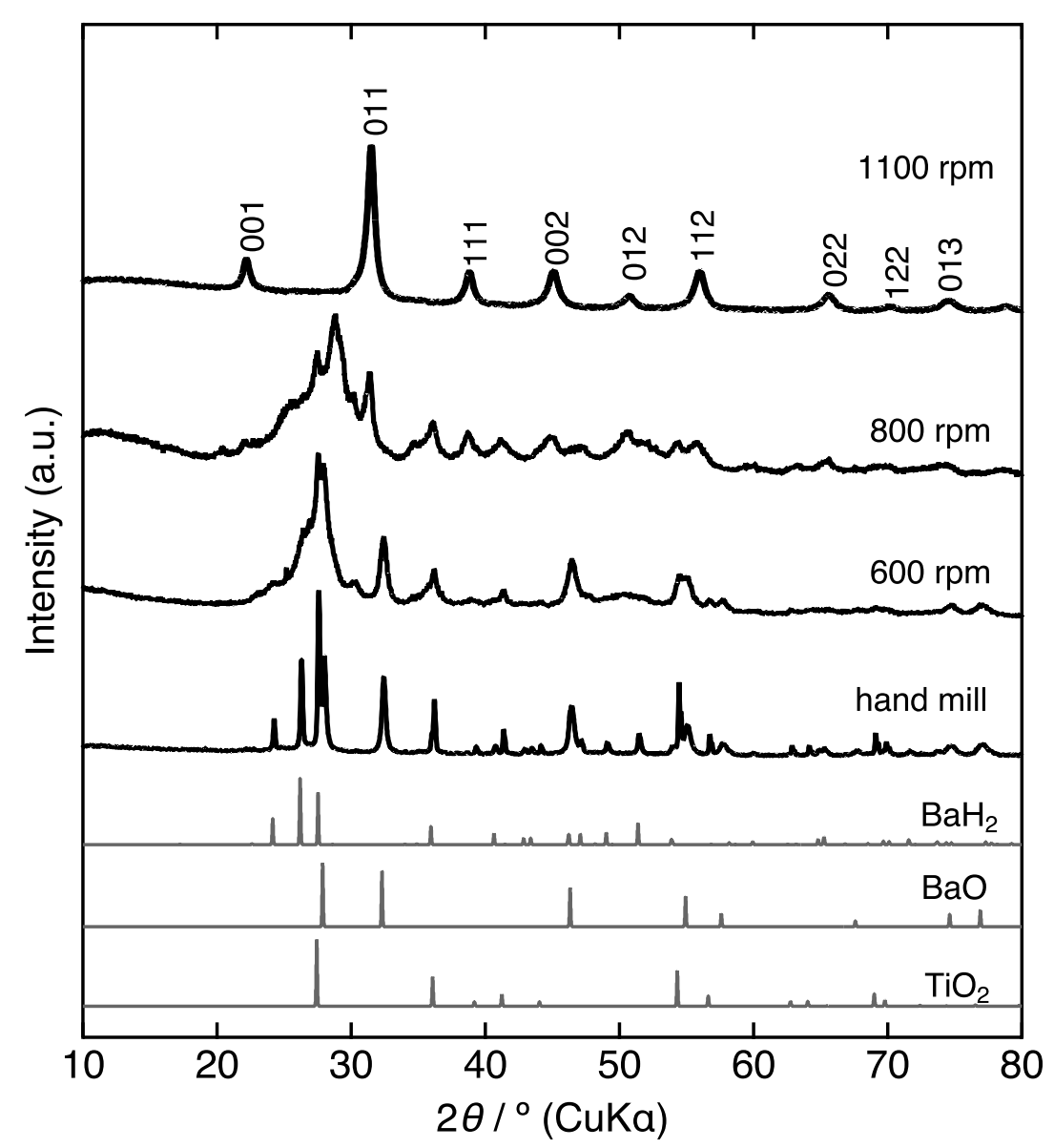

Fig. 1 XRD patterns for preparation composition $\mathrm{BaTiO}_{2.5} \mathrm{H}_{0.5}$ at hand mill, $600 \mathrm{rpm}, 800 \mathrm{rpm}$, and $1100 \mathrm{rpm}$. Selected $h k l$ reflections with relatively intense peaks are indexed. 


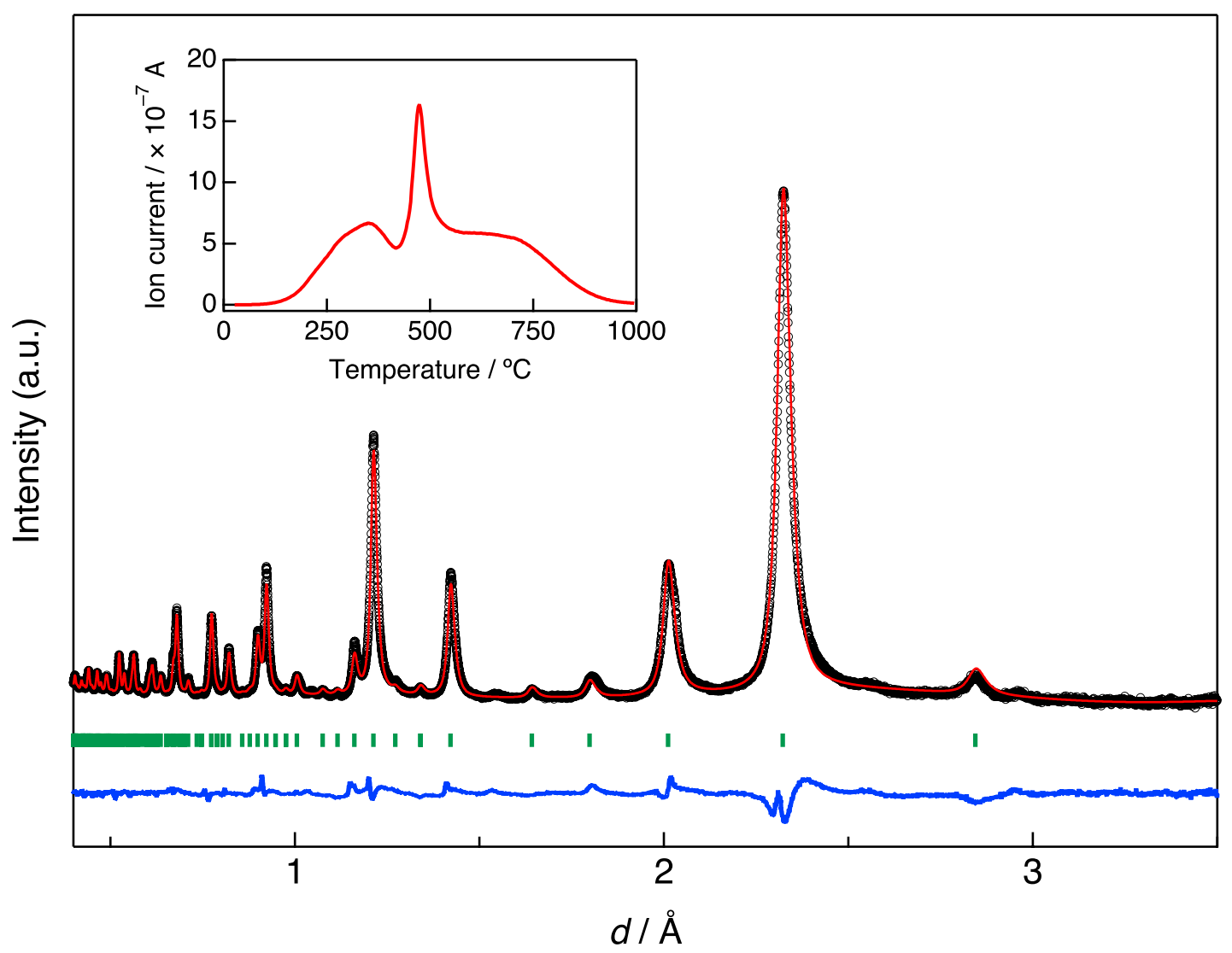

Fig. 2 Rietveld refinements for ND data of $x_{\text {nom }}=0.5$ product. Circles (black) and lines (red) indicate observed and calculated data, respectively. The vertical marks (green) indicate the Bragg reflection positions. The inset shows TDS curve corresponding to $\mathrm{H}_{2}$ desorption upon heating. 

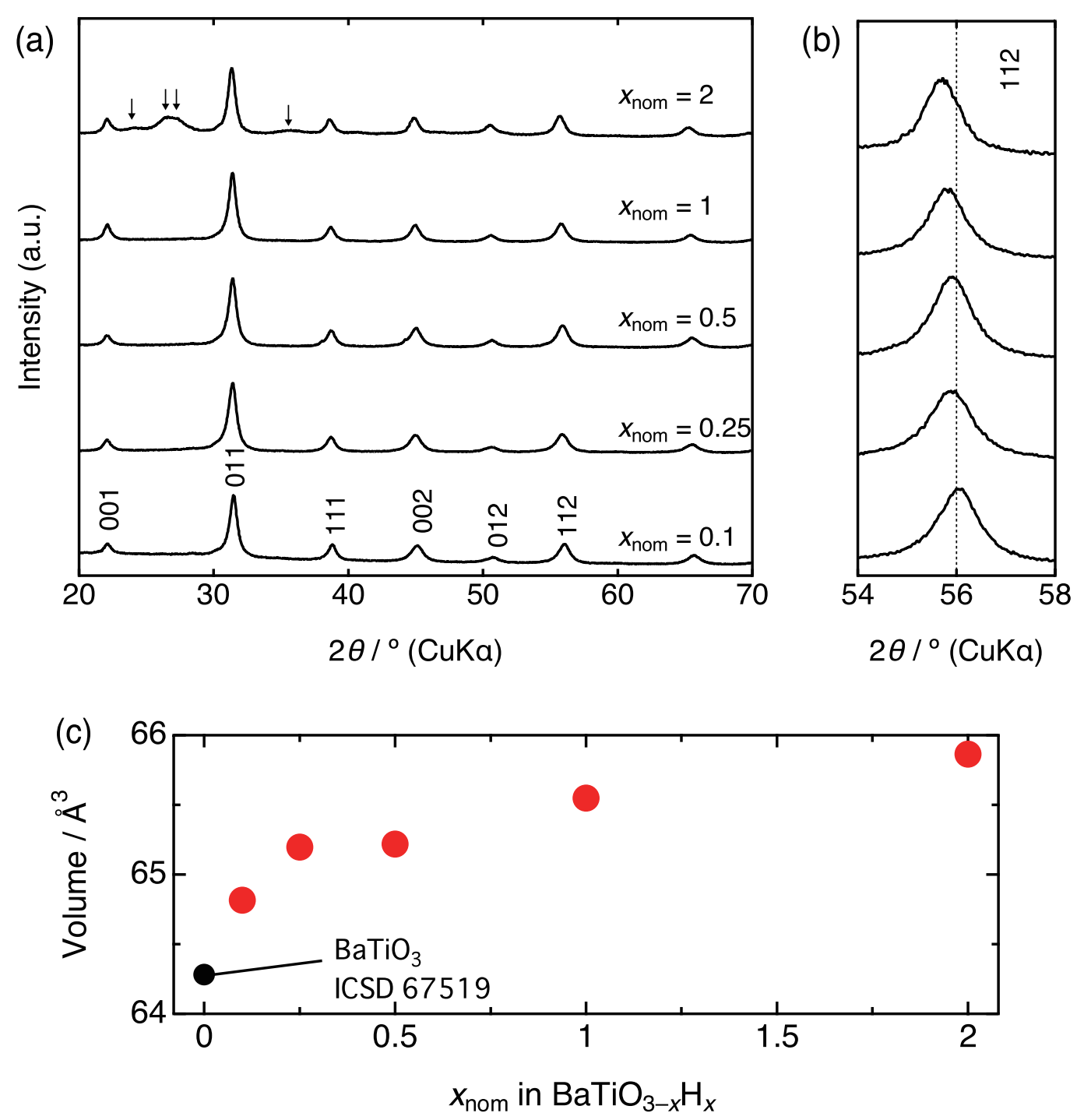

Figure 3 (a) XRD patterns of mechanochemically prepared $\mathrm{BaTiO}_{3-x} \mathrm{H}_{x}$ and (b) the enlarged view at $2 \theta=54-58^{\circ}$ wherein the (112) peak exists. Arrows in $x_{\text {nom }}=2$ indicates peaks derived from $\mathrm{BaH}_{2}$. (c) Volume of the unit cell estimated from lab-XRD depending on $x_{\text {nom }}$ (red circles). Black circle is that of $\mathrm{BaTiO}_{3}$ (ICSD 67519). 
(a)

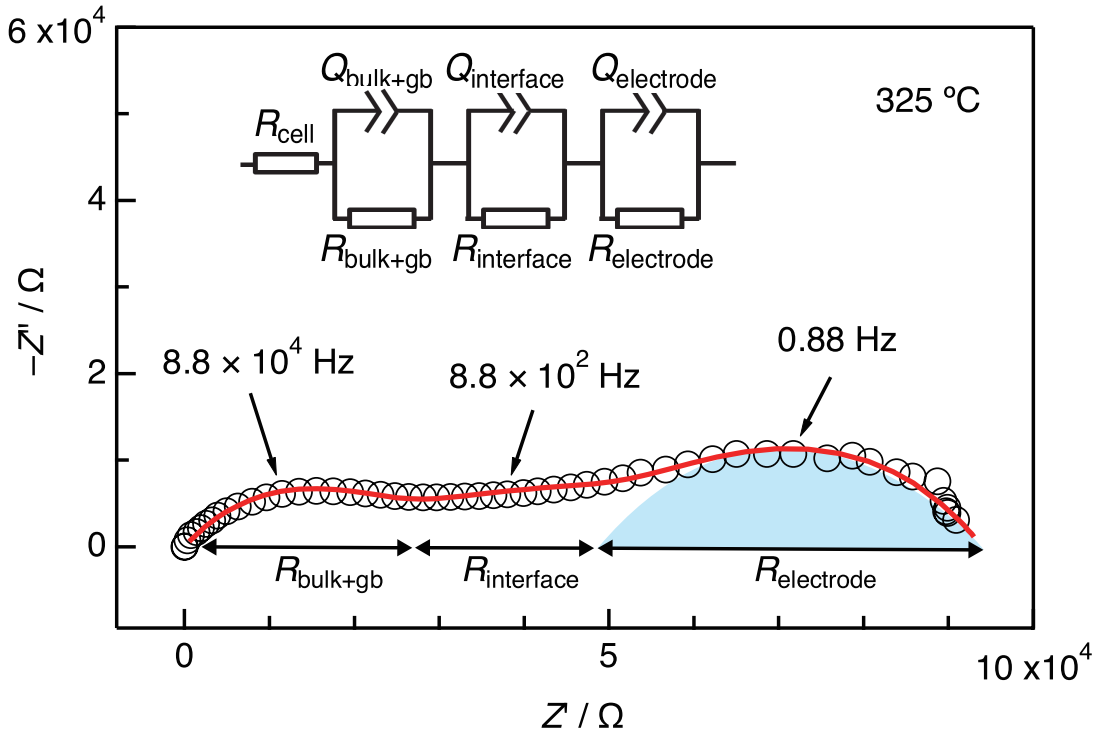

(b)

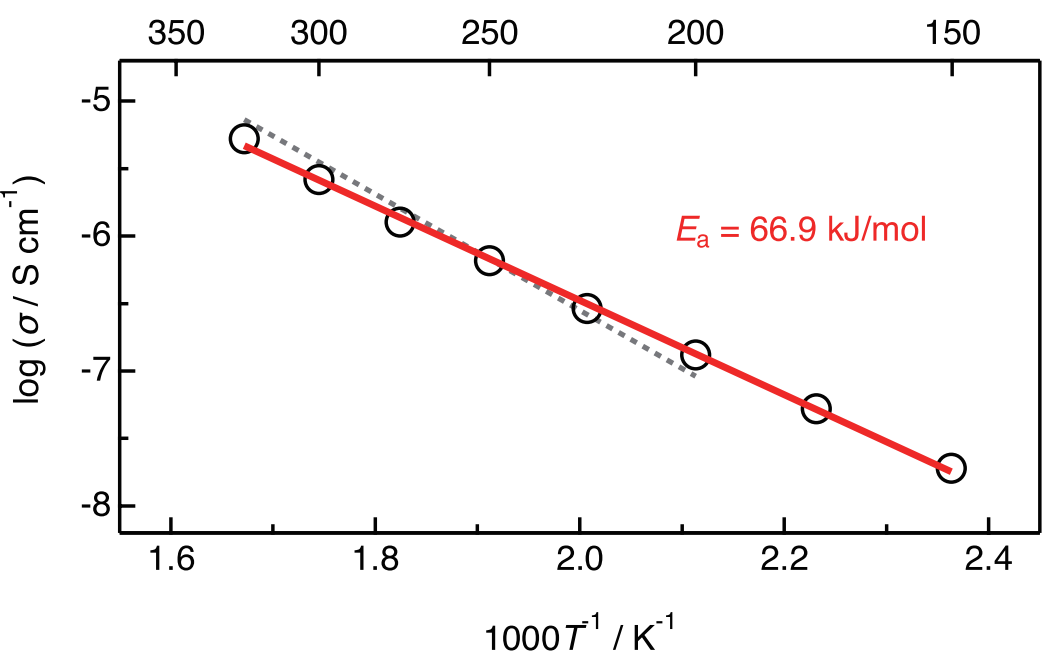

Figure 4 (a) Impedance spectrum of a three-layered symmetric cell composed of electrolyte $\mathrm{LaSrLiH}_{2} \mathrm{O}_{2}$ sandwiched by $\mathrm{BaTiO}_{2.5} \mathrm{H}_{0.5}$ electrodes at $325{ }^{\circ} \mathrm{C}$. The Equivalent circuit used for fitting the spectrum is shown in (a). $R$ and $Q$ represent resistance and constant phase element (CPE). The red solid line is fitting result. (b) Arrhenius plots for the total conductivity of $\mathrm{LaSrLiH}_{2} \mathrm{O}_{2}$ (open circle) and its linear fitting (red solid line). Grey dashed line indicates the reported data using deposited Au electrodes ${ }^{19}$. 